\title{
Crystal Induced Arthropathies: A Pictorial Review
}

Sunita Dhanda* and Swee Tian Quek

Department of Diagnostic Imaging, National University Hospital, 5 Lower Kent Ridge Road, Singapore

*Corresponding author: Dr. Sunita Dhanda MD, FRCR, FRANZCR, Department of Diagnostic Imaging, National University Hospital, 5 Lower Kent Ridge Road, 119074 , Singapore, Tel: +65-90619157; E-mail: sunitadhanda63@gmail.com

Received date: October 21, 2016; Accepted date: October 24, 2016; Published date: October 28, 2016

Copyright: () 2016 Dhanda S, et al. This is an open-access article distributed under the terms of the Creative Commons Attribution License, which permits unrestricted use, distribution, and reproduction in any medium, provided the original author and source are credited.

\section{Crystal Induced Arthropathies}

Crystal induced arthropathies are disorders involving crystal deposition in the articular and peri articular soft tissues with resultant inflammation. Gout (Figures 1 and 2) and calcium pyrophosphate dihydrate (CPPD) crystal deposition disease (Figures 3 and 4) are the two most common types of crystal- deposition arthritis [1]. The diagnosis is primarily based on clinical and laboratory findings, especially synovial fluid analysis [2]. Radiographs can further aid clinicians in establishing the correct diagnosis.

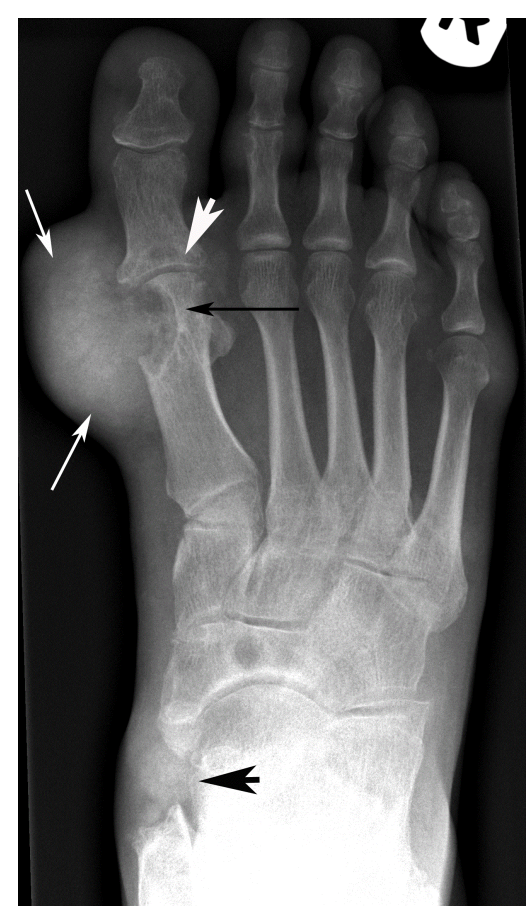

Figure 1: Chronic tophaceous gout. Anteroposterior (AP) radiograph of the right foot shows classic appearance of gouty arthritis at the first metatarsophalangeal joint as a focal eccentric soft tissue swelling due to a tophus (thin white arrow) and welldefined punched out juxta-articular erosion with sclerotic rims and overhanging edges of the underlying first metatarsal head (thin black arrow). Joint space is preserved (thick white arrow). A tophus is also noted at the ankle joint (thick black arrow).

Acute gout most frequently involves the first metatarsophalangeal (MTP) joint at first presentation. However, it has non-specific radiographic features [2]. Chronic gout has a characteristic radiographic appearance with tophus considered to be a hallmark feature [2].

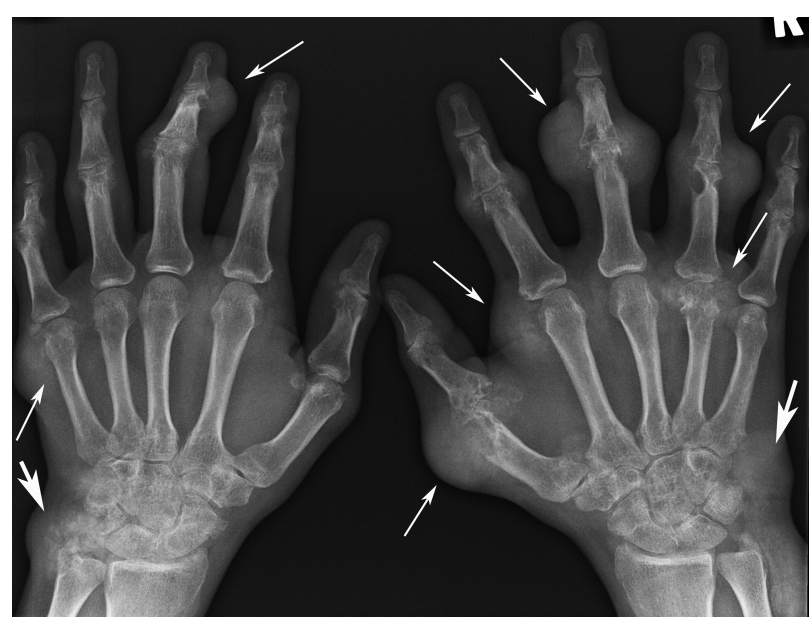

Figure 2: Postero-anterior (PA) radiograph of both the hands shows bilateral asymmetric polyarticular gouty tophi involving the joints of the hands (thin white arrows) and wrists (thick white arrow) and underlying characteristic erosions with no predilection for a specific joint.

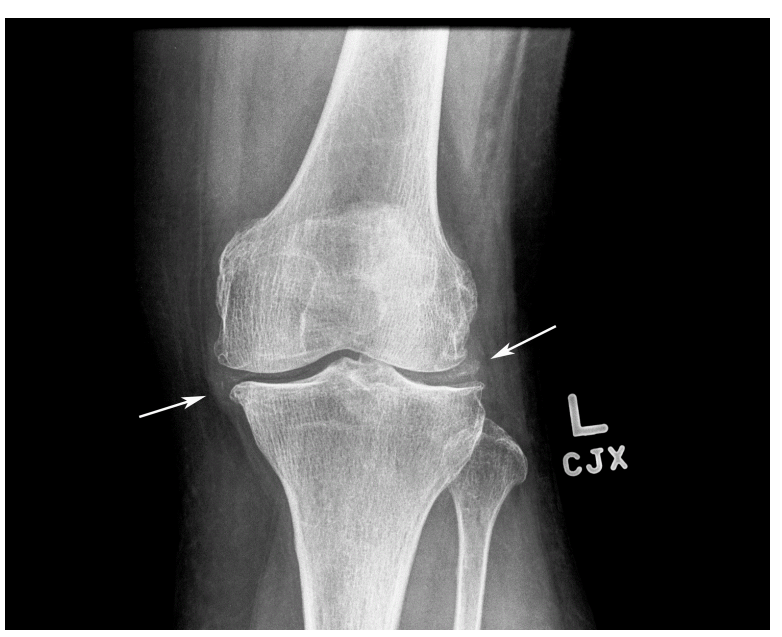

Figure 3: Chondrocalcinosis. AP radiograph of the knee joint in a patient with CPPD arthropathy shows wedge-shaped calcification in the fibrocartilaginous menisci (thin white arrows). 


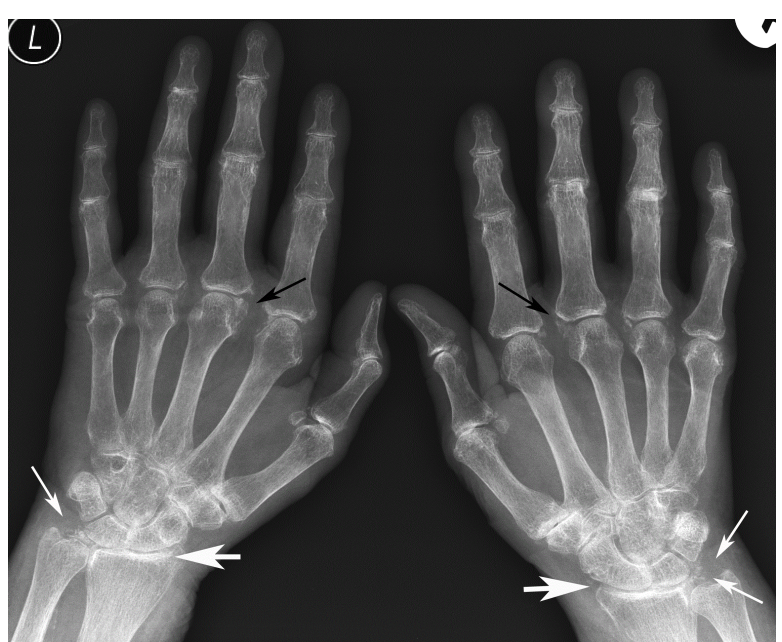

Figure 4: CPPD arthropathy of hands and wrists. PA radiograph shows calcification of the triangular fibrocartilage on both the sides and hyaline cartilage or ligament between the right lunate and the triquetrum (thin white arrows). Capsular calcification is noted at bilateral metacarpophalangeal joints (thin black arrows). There is loss of radioscaphoid and scapholunate joint spaces with subchondral sclerosis and without any erosions (thick white arrows), not the typical distribution for primary osteoarthritis.

Tophi are seen as focal eccentric soft tissue swelling of the affected joints or soft tissues resulting in a "lumpy bumpy appearance" and cause well-defined punched out juxta-articular erosions with sclerotic rims and overhanging edges due to pressure on the adjoining bone (Figure 1). Normal bone mineralisation and joint spaces are preserved till late in the disease. It occurs in asymmetrical polyarticular distribution with involvement of the feet, ankles, knees, hands, and elbows, in decreasing order of frequency (Figure 2) [1,2].

CPPD arthropathy may present as chondrocalcinosis or severe arthropathy. CPPD arthropathy radiographically mimics osteoarthritis showing subchondral sclerosis and osteophyte formation. However, it has a distinctive distribution pattern with knees, hands, and hips involved in decreasing order of frequency and shows uniform loss of joint space [1]. Unlike osteoarthritis, the shoulder and elbow are also involved. Subchondral cysts are more prominent than in osteoarthritis. Normal bone mineralization is preserved. There are no articular erosions $[1,2]$. Chondrocalcinosis, representing deposition of CPPD crystals into fibrous or hyaline cartilage is seen most frequently in the knee (Figure 3), pubic symphysis, and wrist (Figure 4) [1-3]. The arthropathy of the wrist most commonly affects the radiocarpal joint (Figure 4). Scapholunate dislocation may be seen with narrowing of the joint space between the lunate and capitate and resultant "stepladder" configuration. Wrist involvement may result in scapholunate advanced collapse. In the hands, metacarpophalangeal joints are primarily involved with tendency towards calcification of the synovium and capsule around these joints (Figure 4) [1]. Hence, features of joint space loss, subchondral sclerosis, osteophyte formation and cysts in the wrong distribution for primary osteoarthritis should raise a concern for CPPD arthropathy and prompt a search for evidence of articular or periarticular crystal deposition.

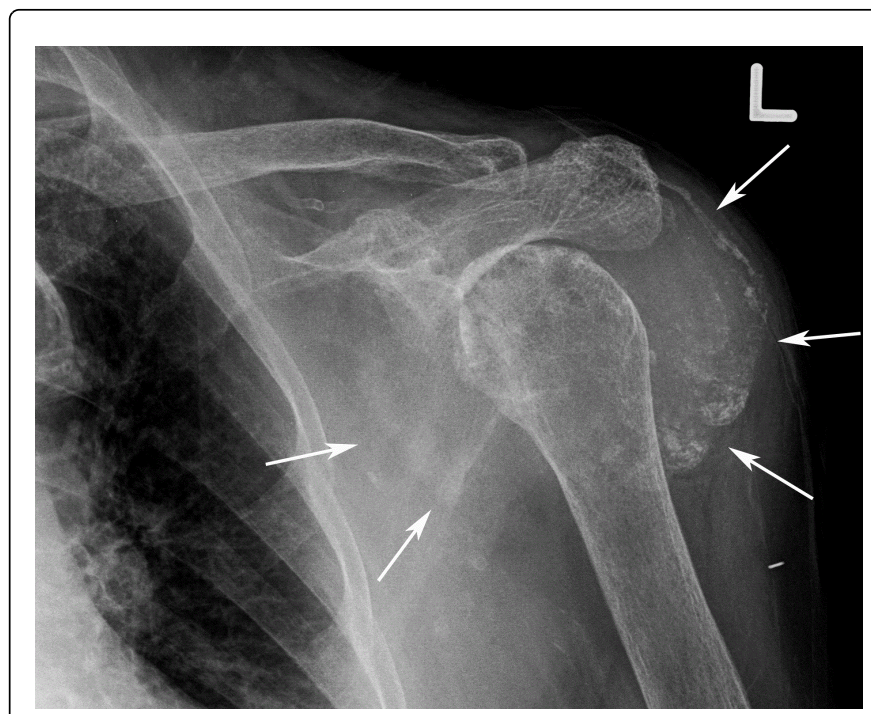

Figure 5: "Milwaukee shoulder" in a patient with HADD. AP radiograph of the left shoulder shows amorphous calcification in the distended subacromial-subcoracoid bursa as well as the axillary recess (thin white arrows) with partial resorption of the humeral head.

Hydroxyapatite deposition disease (HADD) causes periarticular disease in the form of tendinitis or bursitis and rarely a true articular disease. The shoulder is the most common site of calcific tendinitis or bursitis [1]. Rotator cuff tendon calcification may eventually rupture into the bursa and lead to a severe destructive arthropathy known as "Milwaukee shoulder" (Figure 5).

\section{References}

1. Brower AC, Flemming DJ (2012) Arthritis in Black \& White. (3rd edtn). Saunders Elsevier, Philadelphia, PA, USA.

2. Choi MH, MacKenzie JD, Dalinka MK (2006) Imaging features of crystal induced arthropathy. Rheum Dis Clin North Am 32: 427-446.

3. Steinbach LS (2004) Calcium pyrophosphate dihydrate and calcium hydroxyapatite crystal deposition diseases: Imaging perspectives. Radiol Clin North Am 42: 185-205. 